\title{
Design Nonlinear Model-free Neuro-fuzzy ARX Algorithm to Control of System's Temperature
}

\author{
Fatemeh Dehghan Ashkezari ${ }^{1,2}$, Farzin Piltan ${ }^{1}$, Maryam Sarostad $^{1,2}$ \\ and Nasri B. Sulaiman ${ }^{1,3}$ \\ ${ }^{1}$ Intelligent Systems and Robotics Lab, Iranian Institute of Advanced Science and \\ Technology (IRAN SSP), Shiraz/Iran \\ ${ }^{2}$ Department of Computer Engineering, Yazd University, Yazd/Iran \\ ${ }^{3}$ Department of Electrical and Electronic Engineering, Faculty of Engineering, \\ University Putra Malaysia, Malaysia \\ Piltan_f@iranssp.org,www.iranssp.org/english
}

\begin{abstract}
Control of system's temperature is one of the active research areas in field of energy consumption. In this research we have following objectives: temperature data collection from system, intelligent system identification based on neuro-fuzzy Auto Regressive eXternal model input (ARX) methodology and design a nonlinear controller to fixed a temperature and improve the energy efficiency. To control the system's temperature, data collection and data analysis are two most important factors. After data collection, system identification plays an important role to control systems. Neuro-fuzzy ARX is one of the significant method to system modeling. In this research, the number of training data is less than 100 samples. To control of system's temperature, Pulse Width Modulator $(P W M)$ is used by the nonlinear model free controller to fixed system's temperature. However tuning the system's temperature is extremely important but the other important factors is the rate of tuning the temperature. Nonlinear model free robust functional based is used in this research.
\end{abstract}

Keywords: nuro-fuzzy system modeling, ARX identified algorithm, pulse width modulator (PWM) algorithm, nonlinear functional based model free control techniques, robust algorithm.

\section{System Modelling}

Temperature is used to comparative between hot and cold environment and used to measure of material's quality. Most of researchers have limitation to measure the temperature, therefore intelligent modeling one of the best technique for this type of data. In this research, change the voltage caused to duration in temperature. Pulse Width Modulation (PWM) used to tuning the rate of temperature changes. Figure 1 shows the input/output system's data [1-4]. 

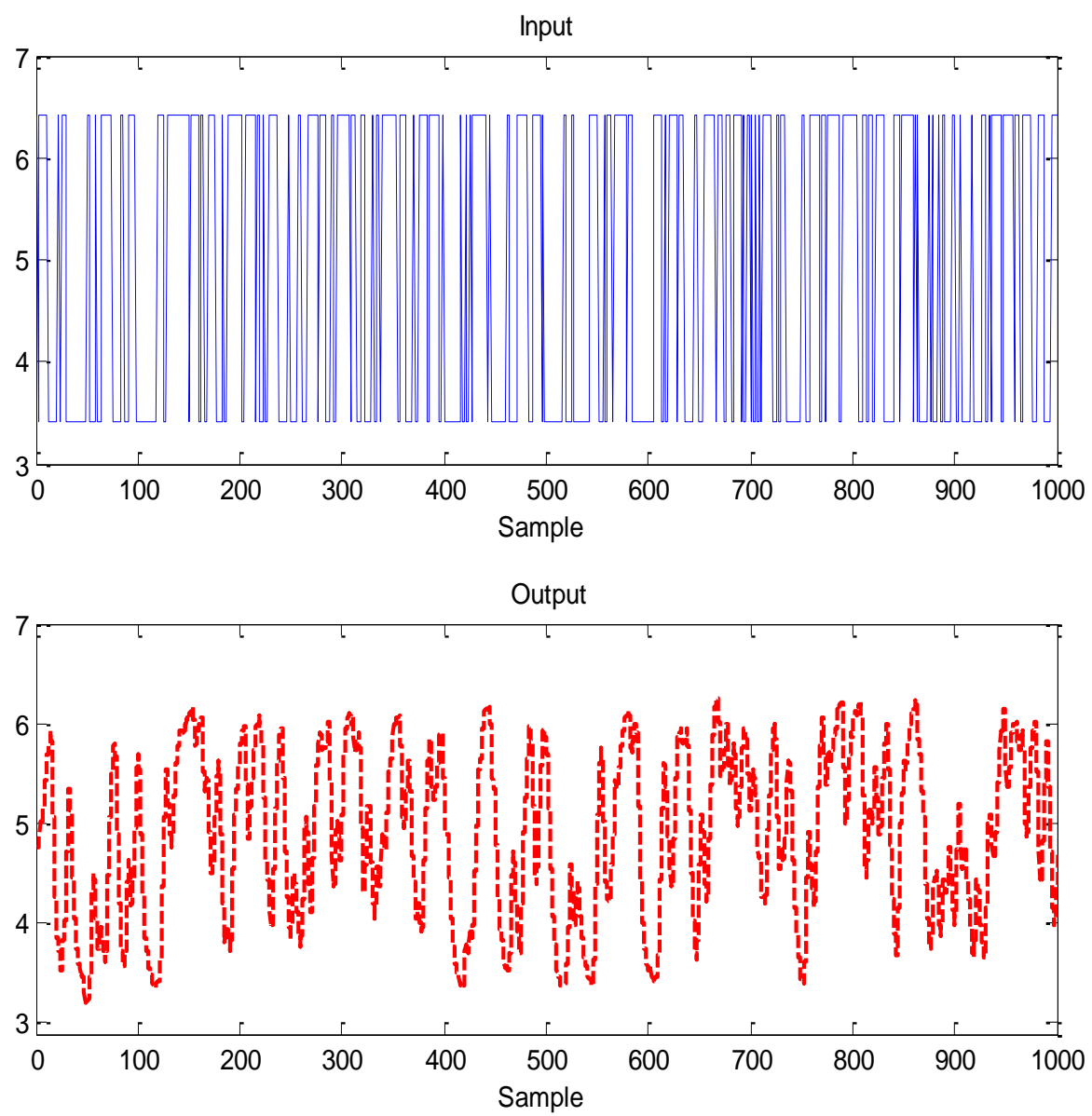

Figure 1. Data Collection (Voltage/Temperature)

Regarding to Figure 1, to tuning the temperature between three to six degrees of siliceous the voltage must change based on PWM. The rate of temperature changes shows in the Figure 2. Regarding to Figures 1 and 2, temperature and rate of temperature have fluctuations. According to Figure 1, we have 1000 samples but we use 100 samples for data training by neuro fuzzy technique. 


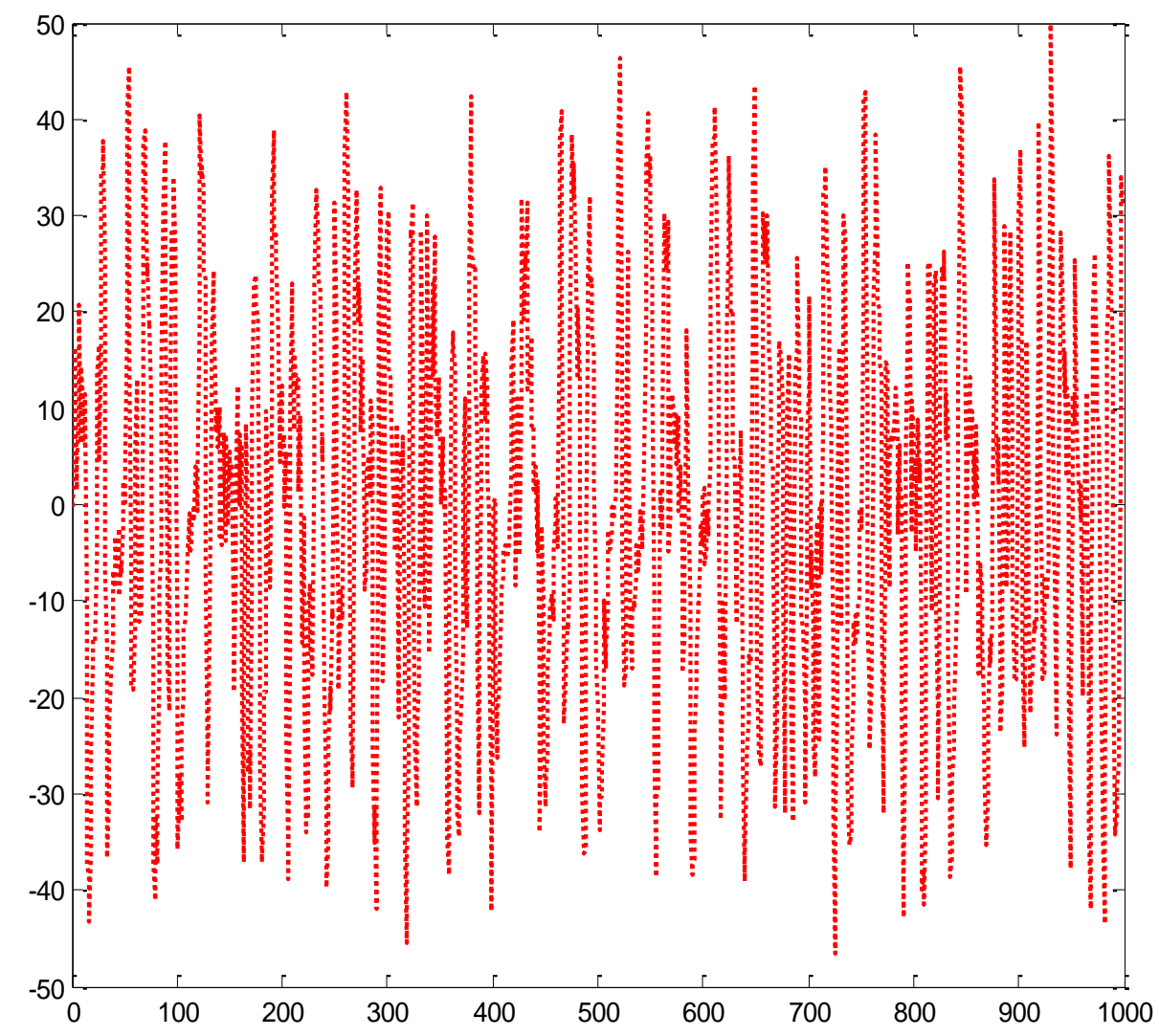

Figure 2. Rate of Temperature Changes

Based on neuro-fuzzy algorithm this design has one input/one output. In this research we defined 7 bell shape rule base. Figure 3 shows the input/output rule-base.

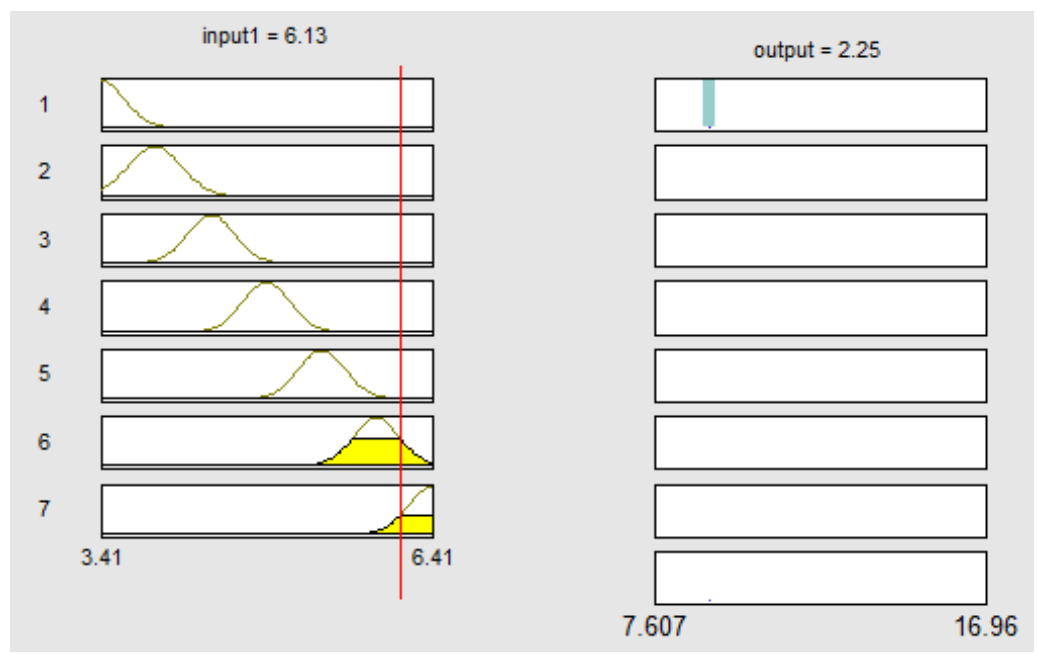

Figure 3. Input/Output Rule Base

Based on Figure 3, the rate of input duration is [3.41-6.41] and the rate of output duration is [7.607-16.96]. The input/output surface viewer shows in Figure 4. 


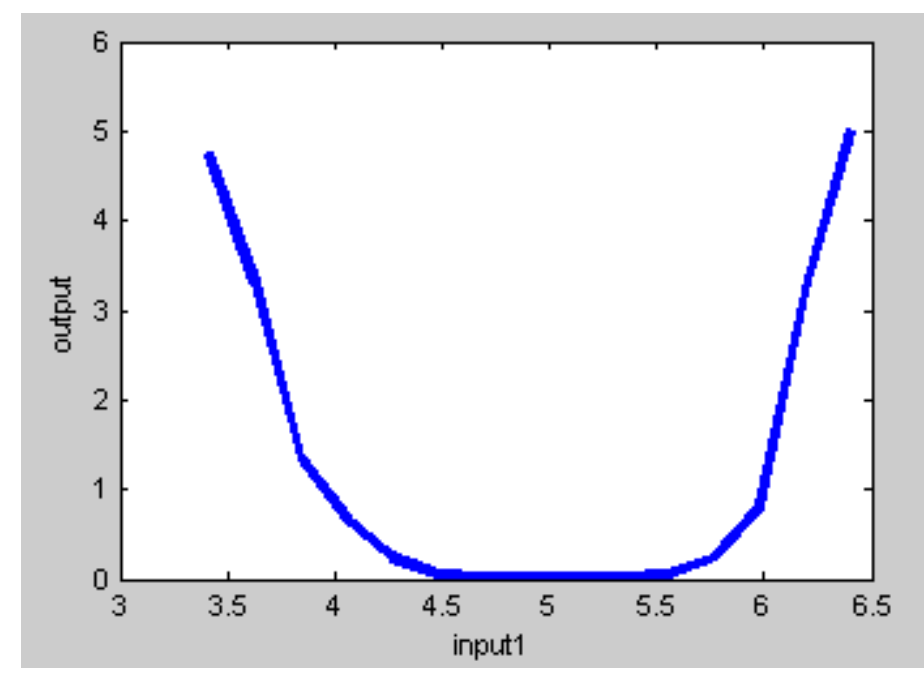

Figure 4. Surface Viewer

According to this design, the training data and error shows in Figure 5.

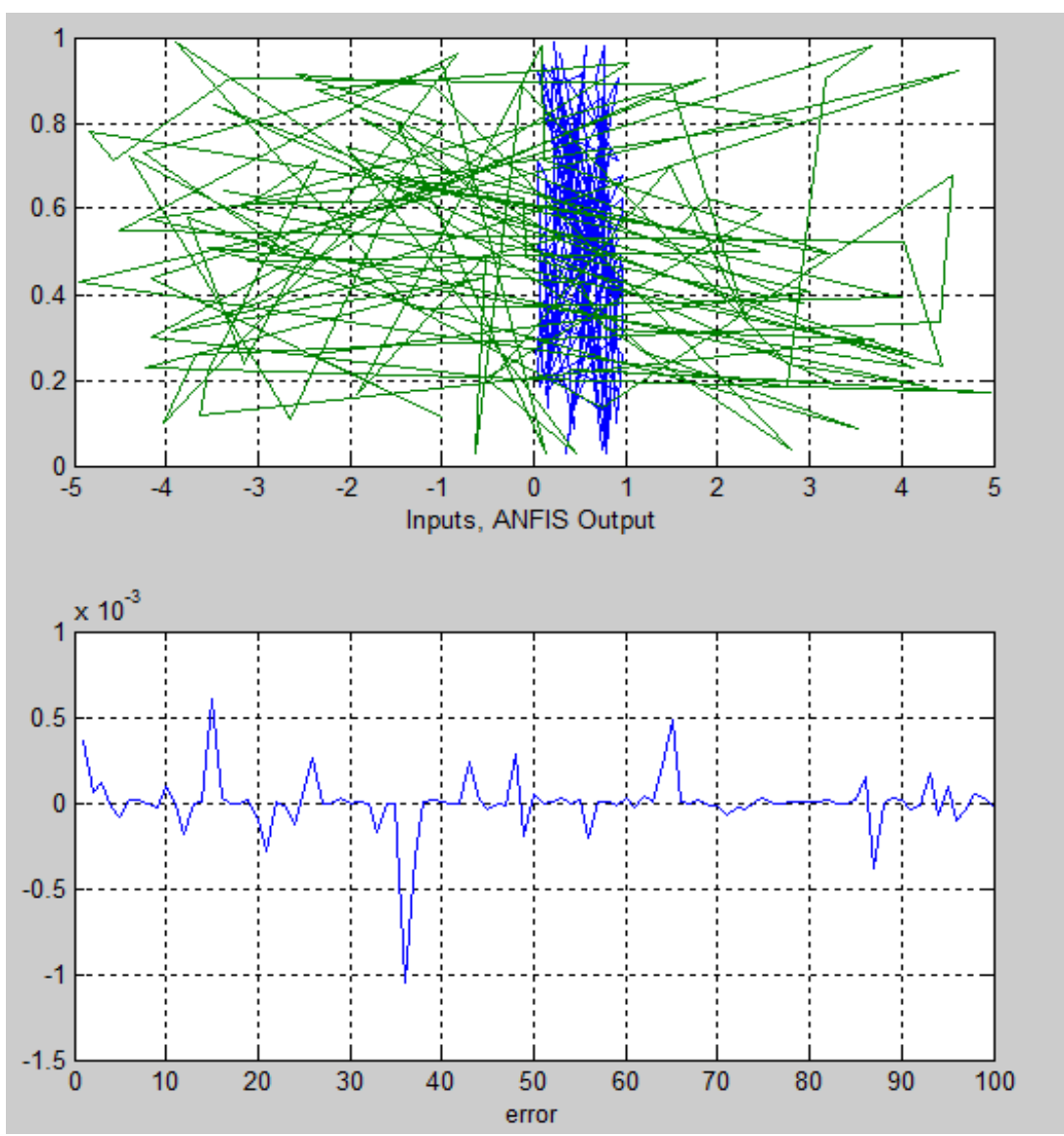

Figure 5. Input/Output ANFIS and Training Error

According to Figure 5, the rate of data training is great in this design. The average of testing error in this design is 0.00036065 . The ANFIS testing data shows in Figure 6. Regarding to this Figure the rate of error is near to the zero. 


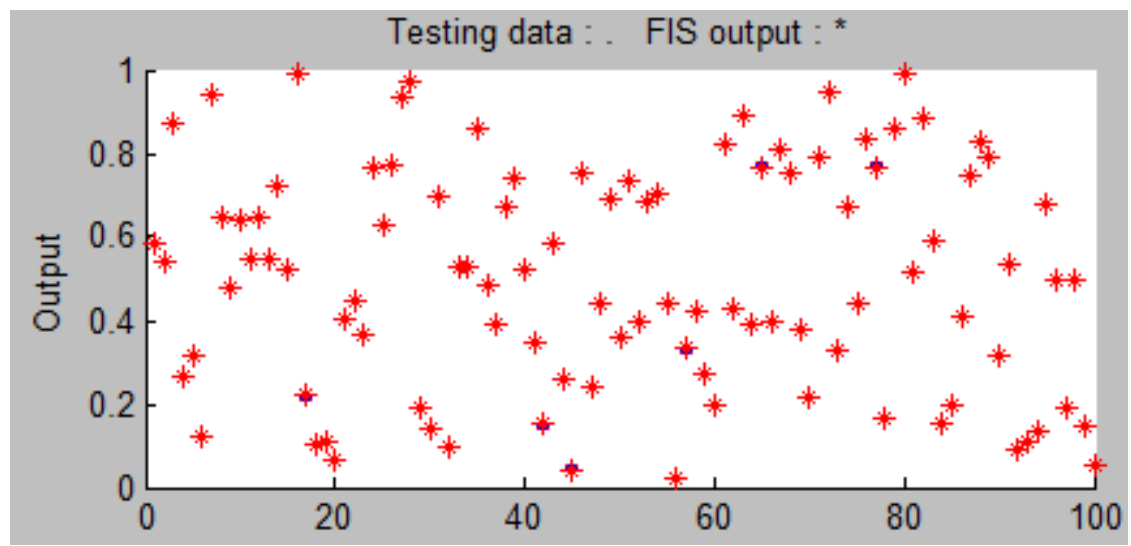

Figure 6. ANFIS Testing Data

After modeling system's temperature based on ANFIS, ARX methodology is used for system identification. To modeling the position system based on ARX theory, the general input-output form is:

$$
A(q) y(t)=\frac{B(q)}{F(q)} u(t-n k)+\frac{C(q)}{D(q)} e(t)
$$

the five polynomials $A(q), B(q), C(q), D(q)$ and $F(q)$ are introduced and the state $A$ is:

$$
A(q)=\left[\begin{array}{llllll}
1 & a_{1} & a_{2} & a_{3} & \ldots & a_{n}
\end{array}\right]
$$

The most used model structure is the simple linear difference equation:

$$
\begin{aligned}
y(t)+a_{1} y(t-1) & +a_{2} y(t-2)+\cdots+a_{n a} y(t-n a) \\
& =b_{1} u(t-1)+b_{2} u(t-2)+\cdots \\
& +b_{n b} y(t-n k-n b+1)
\end{aligned}
$$

which relates the current output $y(t)$ to a finite number of past outputs $y(t-k)$ and inputs $u(t-k)$. To ARX modeling we have [4-5]:

$A(q) y(t)=B(q) u(t-n k)+e(t)$

Assuming the signals are related by a linear system, the relationship can be written

$$
y(t)=G(q) U(t)+e(t)
$$

where $q$ is the shift operator and $\mathrm{G}(\mathrm{q}) . \mathrm{U}(\mathrm{t})$ is short for:

$$
\mathrm{G}(\mathrm{q}) \mathrm{U}(\mathrm{t})=\sum \mathrm{g}(\mathrm{k}) \cdot \mathrm{U}(\mathrm{t}-\mathrm{k})
$$

and

$$
G(q)=\sum g(k) q^{-k}, q^{-1} u(t)=u(t-1)
$$


There are two problems concerning to system identification modeling 1) highly nonlinear system and 2) the techniques of parameter identification. The parameters of the ARX model structure is:

$$
A(q) y(t)=B(q) u(t)+e(t)
$$

The order in ARX modeling is:

$$
\mathrm{nn}=[\mathrm{na} \mathrm{nb} \mathrm{nk}]
$$

Where $n a$ is output order in ARX, $n b$ is input order in ARX and $n c$ is delay order.

In this system if $n n=[2,2,3]$ the system identification is as Figure 7 .

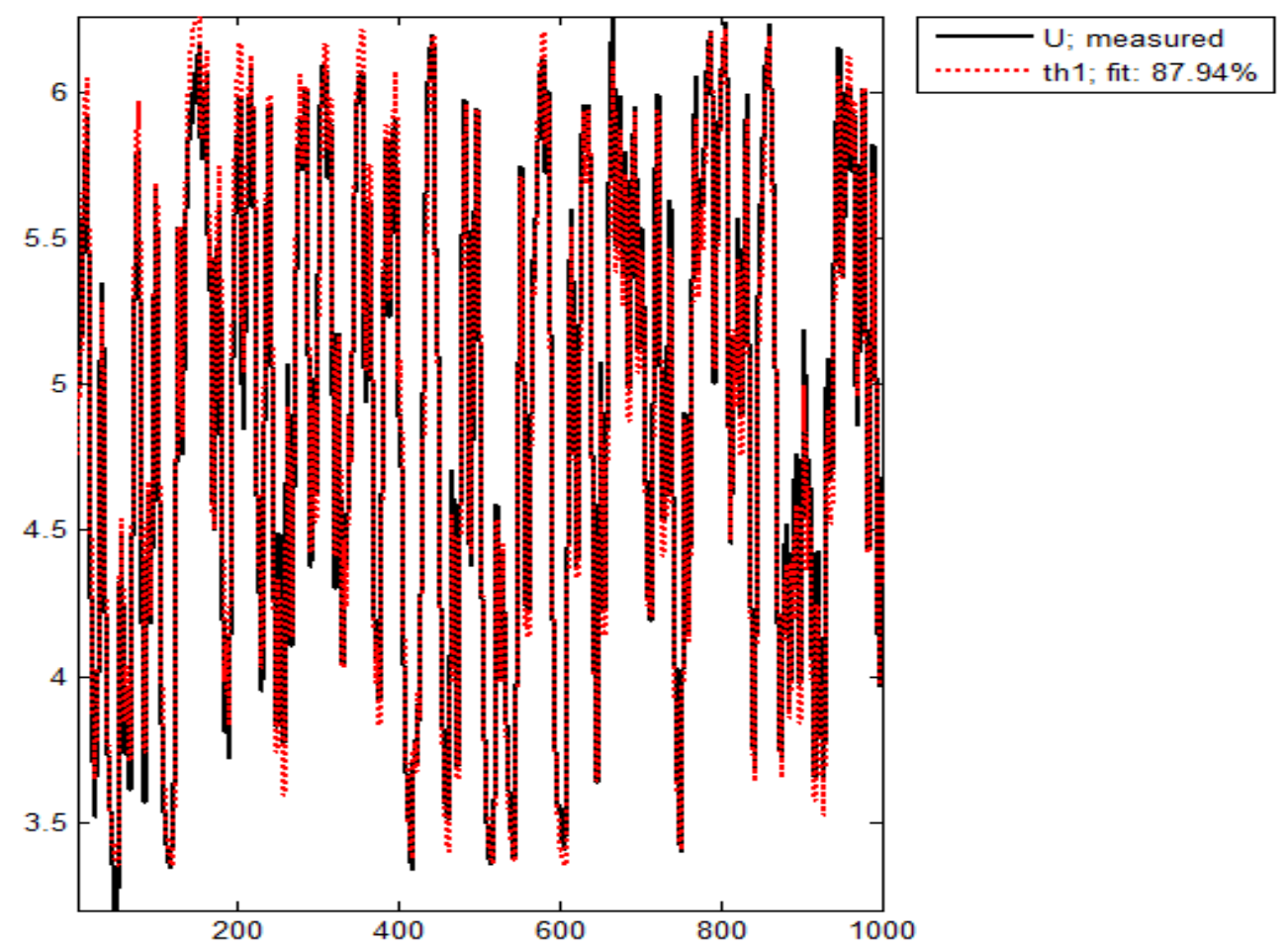

Figure 7. Fitting Test Compare between ARX Identification and Output, 87.94\% Fitting Test

Regarding to Figure 7, however system's fitting rate is $87.94 \%$ but it has error. Figure 8 shows the error rate of ARX identification. 


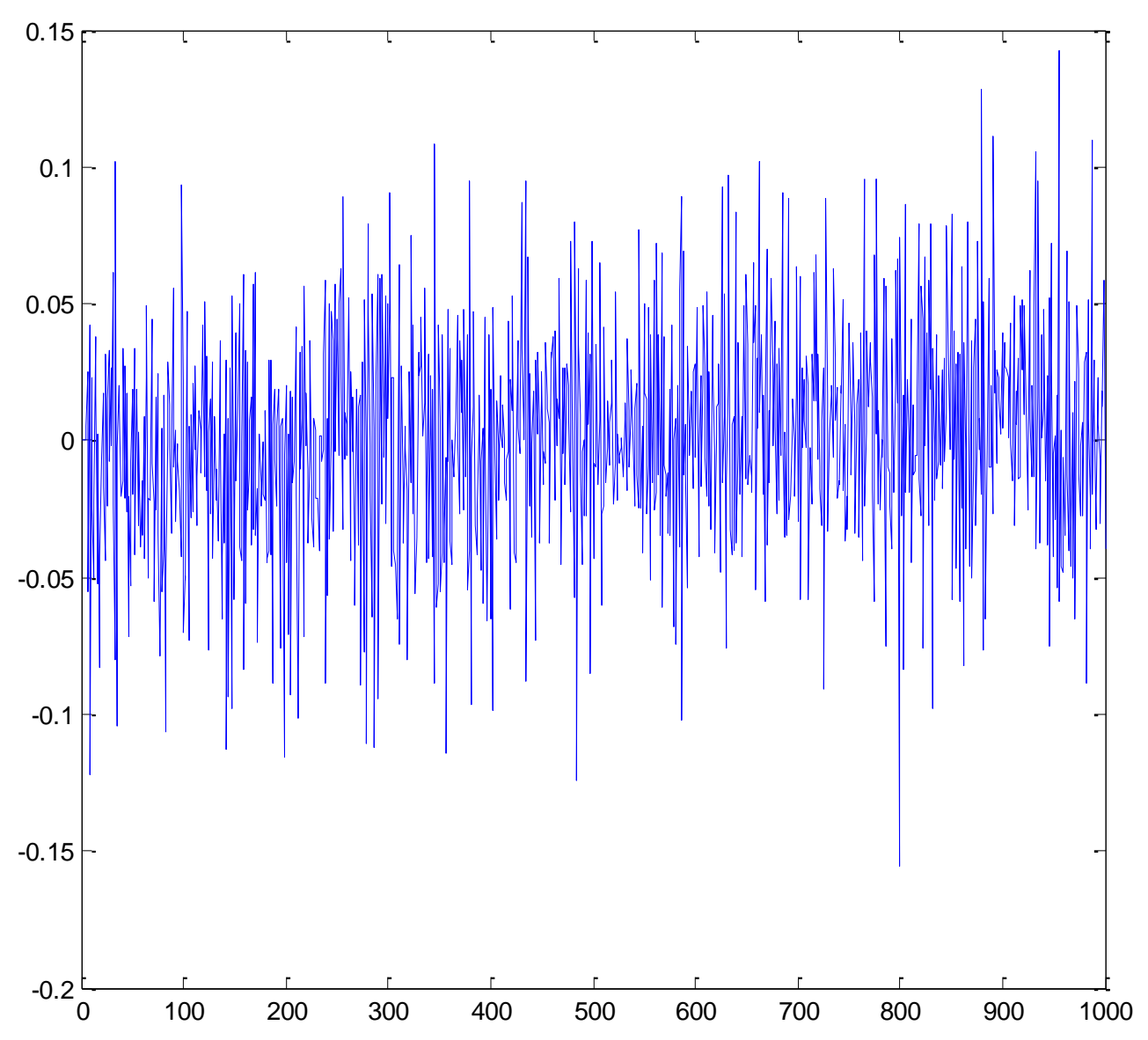

Figure 8. Error Compare between ARX Identification and Real System

Regarding to Figure 7, the Discrete-time IDPOLY model is:

$$
\begin{aligned}
& A(q) y(t)=B(q) u(t)+e(t) \\
& \begin{aligned}
A(q)=1- & 1.283( \pm 0.01084) q^{-1} \\
& +0.3966( \pm 0.00968) q^{-2}
\end{aligned} \\
& B(q)=0.0665( \pm 0.001064) q^{-3}+0.04468( \pm 0.001645) q^{-4}
\end{aligned}
$$

Based on the system identification the loss function is 0.00164908 and final predictive error is 0.00166228 . The continuous time IDPOLY model is:

$$
\begin{aligned}
& A(s) y(t)=B(s) u(t)+C(s) e(t) \\
& A(s)=s^{2}+0.9248 s+0.1768 \\
& B(s)=0.003809 s+0.1729 \\
& C(s)=s^{2}+2.143 s+1.555
\end{aligned}
$$


The Z-transform of this system is:

$$
H(Z)=\frac{0.0665 Z+0.0447}{Z^{4}-1.2829 Z^{3}+0.3966 Z^{2}}
$$

The S-transform of this system is:

$$
H(S)=\frac{(0.0038 S+0.1729)}{S^{2}+0.9248 S+0.1768}
$$

According to above continuous functions the step response of system's temperature shows in Figure 9.

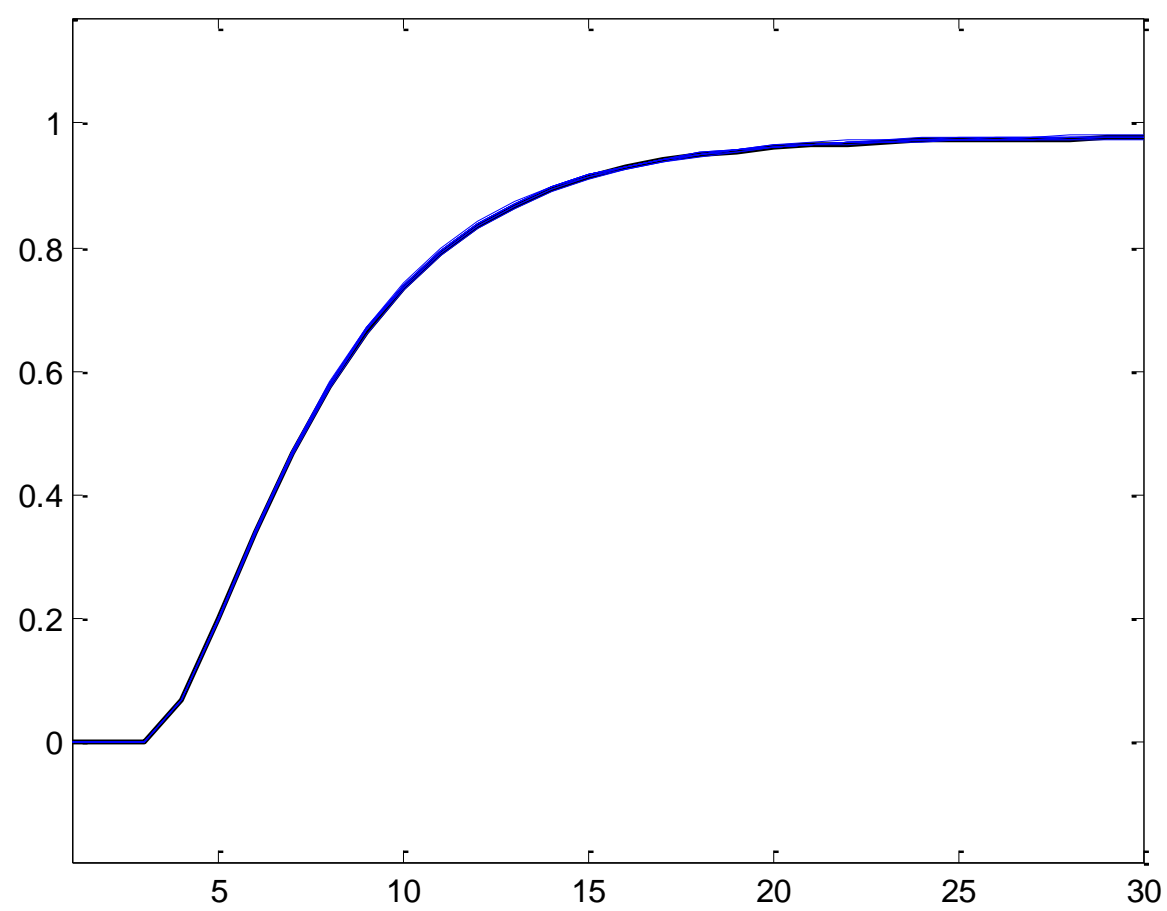

Figure 9. Step Response with ARX Identification and Real System

\section{Design Control Algorithm}

The combination of proportional (P) component, integral (I) component with a derivative (D) controller offered advantages in each case. This type of controller has rapid response to the input deviation, the exact control at the desired input as well as fast response to the disturbances. The PID controller takes the error between the desired input variable and the actual input variable to control the linear and nonlinear system. A proportional-derivative integral control system can easily be implemented. This method does not provide sufficient control for systems with time-varying parameters or highly nonlinear systems. After system identification in this research, our system is second order and highly nonlinear so this controller cannot used alone. The formulation of PID controller is [5-6]:

$U_{P I D}=K_{p} \times e+K_{i}\left(\frac{1}{T} \int e . d t\right)+K_{v}\left(\frac{d e}{d t}\right)=K_{p} \times e+K_{i} \sum e+K_{v} \dot{e}$ 
To improve the result in PID controller, the nonlinear model-free controller is introduced. In this type of controller integral type is add to PID controller and caused to improve the error rate and reduce some parameters such as overshoot. The formulation of new methodology controller is:

$U_{N E W \text { Method }}=\left[K_{p} \times E(s)+\frac{K_{i}}{S} E(s)+S \cdot K v \cdot E(s)\right] \times \frac{K_{i 2}}{S} \cdot E(s)$

$U_{N E W \text { Method }}=\left[K_{p} \times \frac{K_{i 2}}{S} \cdot E^{2}(s)+\frac{K_{i K_{i 2}}}{S} E(s)+S \cdot K v \cdot E(s)\right] \times \frac{K_{i 2}}{S} \cdot E(s)$

In certain condition, regarding to Figure 10, the rise-time in open loop thermal system is high (11.3 second). In PID controller we can reduce the rise-time from 11.3 second to 0.169 second. It means that, we can solve the rise-time challenge but we have the other challenge in PID controller for thermal system. Overshoot is the main challenge of PID controller, in this research we have $12 \%$ overshoot. To reduce the rise-time as well overshoot, the nonlinear model-free $P I^{2} D$ controller is selected in this research. Regarding to Figure 10, this method reduce rise-time from 11.3 second to about 0.2 seconds and reduce the overshoot from $12 \%$ to $1.2 \%$.

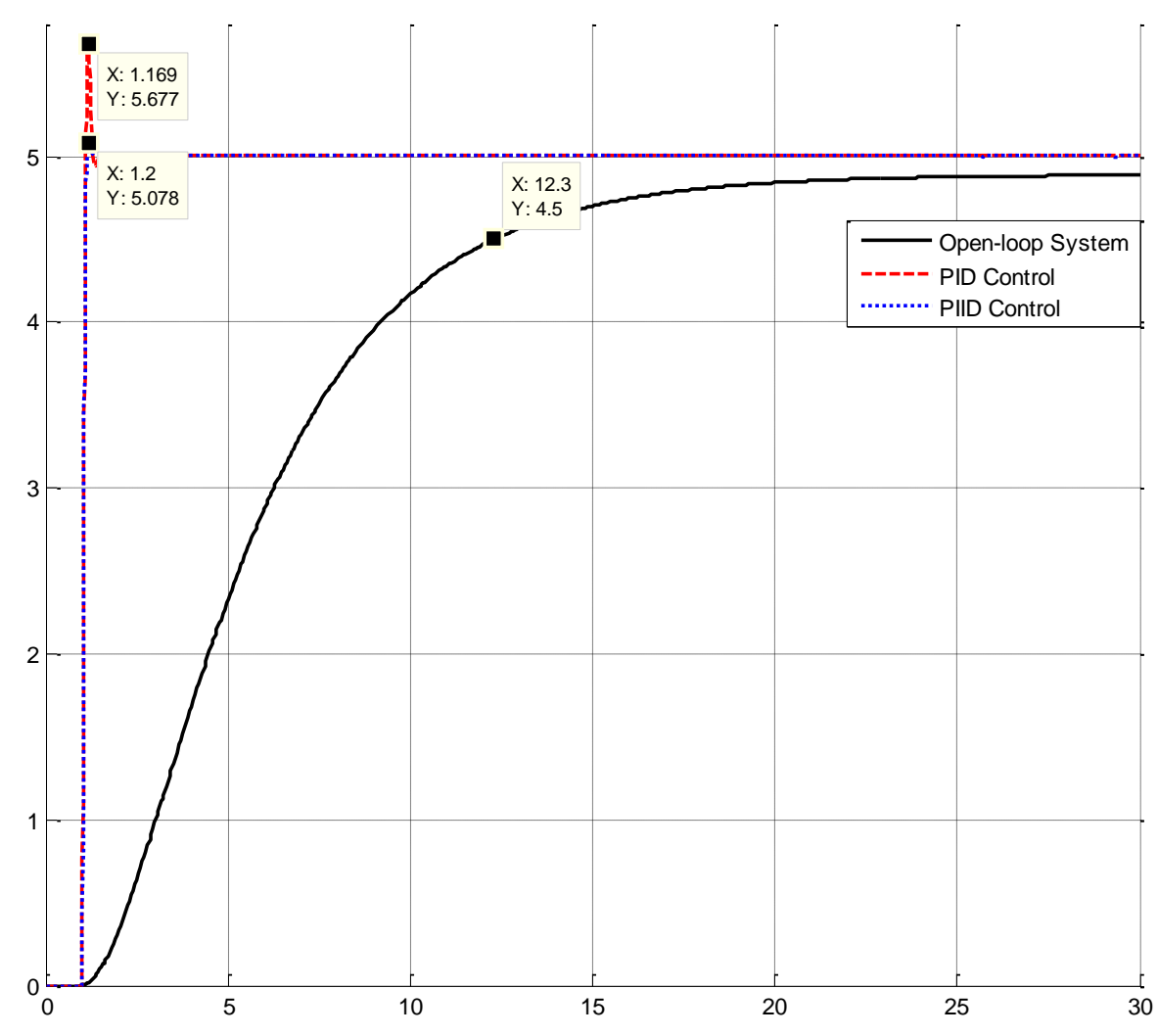

Figure 10. Trajectory following: rise-time, overshoot test in open-loop thermal system, PID controller and nonlinear model-free $P I^{2} D$ controller

Figure 11, shows the error performance comparison between PID controller and $P I^{2} D$ controller. Regarding to the following graph, the error in PID controller is about 
0.0015and in $P I^{2} D$ controller is about 0.0019. PID error performance has undershoot compare with $P I^{2} D$ controller.

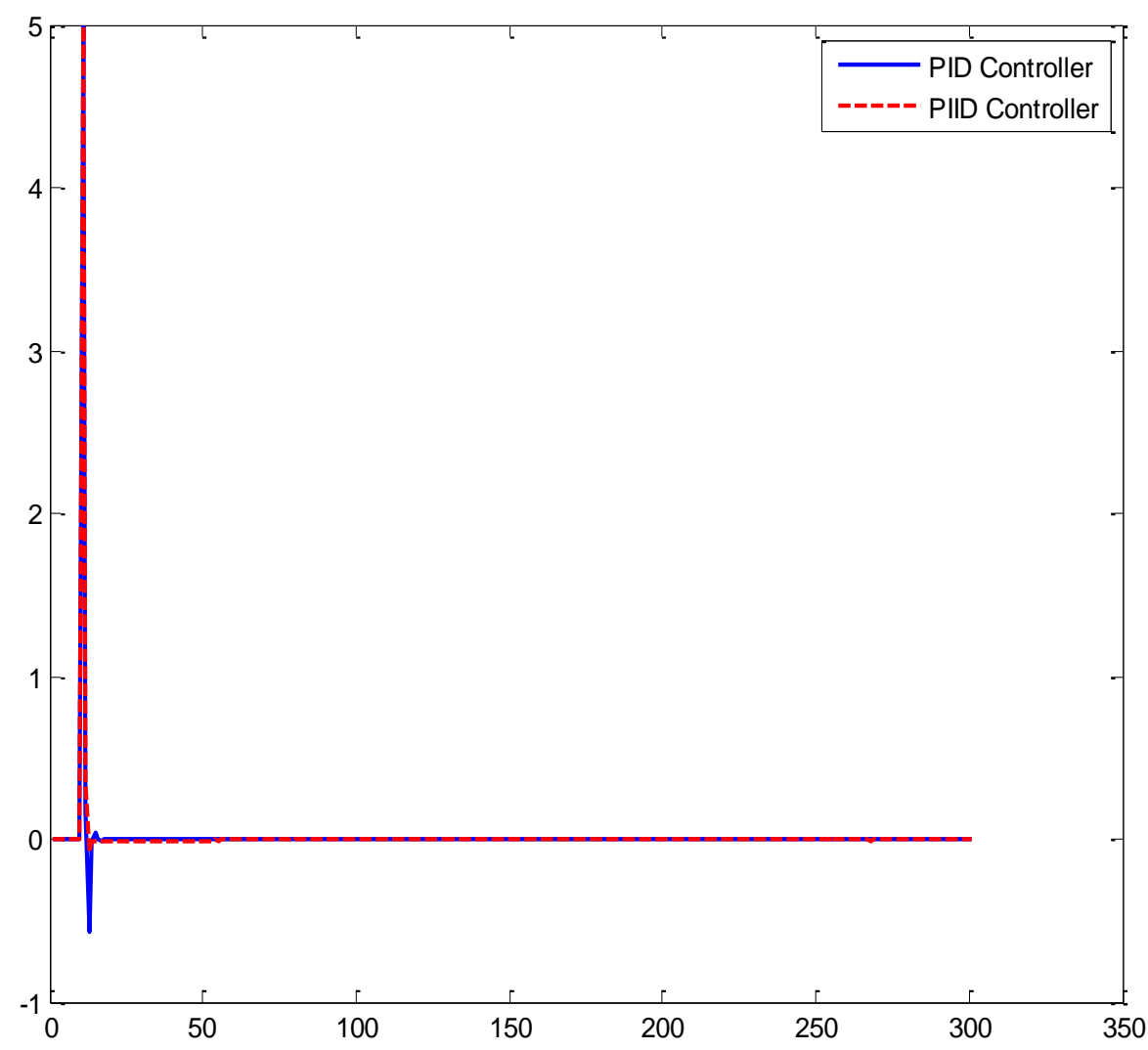

Figure 11. Error Performance: PID Controller and Nonlinear Model-free $\boldsymbol{P I}^{2} \mathrm{D}$ Controller

\section{Conclusion}

The main objectives in this research are: ARX neuro-fuzzy system modeling identify and nonlinear model-free control. For thermal systems, modeling and system identification are important challenges. To solve this challenge, neuro-fuzzy data training is selected to predict the system's transfer function with minimum input data. After applied this method, the average rate of error system modeling is 0.00036065 . After modeling thermal system based on neuro-fuzzy technique, ARX system identification is used to find the Laplas transfer function. After neuro-fuzzy ARX system identification the real system prediction rate is about $87.94 \%$ and final predictive error is 0.00166228 . Two types controller methodology are used to reduce the thermal system rise-time, PID controller and $P I^{2} D$ controller. The nonlinear $P I^{2} D$ controller reduces the rise-time from 11.3 second to about 0.2 seconds and reduces the overshoot from $12 \%$ to $1.2 \%$. Regarding to this research, industrial thermal system is neuro-fuzzy modeling, ARX identifying, and nonlinear model free $P I^{2} D$ control. Based on this method we can system modeling with less than 100 samples. 


\section{Acknowledgment}

This work was supported by the Iranian Institute of Advance Science and Technology Program of Iran under grant no. 2016-Persian Gulf-3.

\section{Project Title: System Identification and Control}

Iranian center of Advance Science and Technology (IRAN SSP) is one of the independent research centers specializing in research and training across of Control and Automation, Electrical and Electronic Engineering, and Mechatronics \& Robotics in Iran. At IRAN SSP research center, we are united and energized by one mission to discover and develop innovative engineering methodology that solve the most important challenges in field of advance science and technology. The IRAN SSP Center is instead to fill a long standing void in applied engineering by linking the training a development function one side and policy research on the other. This center divided into two main units:

- Education unit

- Research and Development unit

\section{Please follow IRANSSP research and training group: http://iranssp.org/english/}

\section{References}

[1] M. A. Jami ‘in, J. Hu, M. H. Marhaban, I. Sutrisno and N. B. Mariun, "Quasi-ARX neural network based adaptive predictive control for nonlinear systems", IEEJ Transactions on Electrical and Electronic Engineering, vol. 11, no. 1, (2016), pp. 83-90.

[2] A. Sar and A. Kural, "Modeling and ARX identification of a quadrotor MiniUAV", In 2015 9th International Conference on Electrical and Electronics Engineering (ELECO), IEEE, (2015) November, pp. 1196-1200.

[3] A. Hartmann, J. M. Lemos, R. S. Costa, J. Xavier and S. Vinga, "Identification of switched ARX models via convex optimization and expectation maximization", Journal of Process Control, vol. 28, (2015), pp. 9-16.

[4] F. D. Rincón, G. A. Le Roux and F. V. Lima, "A novel ARX-based approach for the steady-state identification analysis of industrial depropanizer column datasets", Processes, vol. 3, no. 2, (2015), pp. 257-285.

[5] M. Folgheraiter, "A combined B-spline-neural-network and ARX model for online identification of nonlinear dynamic actuation systems", Neurocomputing, vol. 175, (2016), pp. 433-442.

[6] B. Siciliano and O. Khatib, Springer handbook of robotics: Springer-Verlag New York Inc, (2008).

\section{Authors}

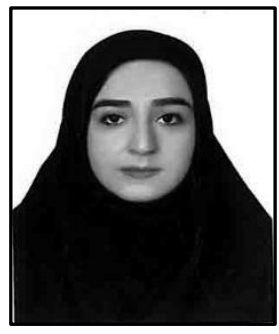

Fatemeh Dehghan Ashkezari, is currently working as a research assistant in Control and Robotic Lab at the institute of advanced science and technology, IRAN SSP research and development Center. Her current research interest is in the area of system identification. 


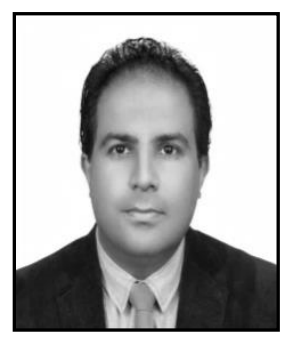

Farzin Piltan, is an outstanding scientist in the field of Electronics and Control engineering with expertise in the areas of nonlinear systems, robotics, and microelectronic control. Mr. Piltan is an advanced degree holder in his field. Currently, Mr. Piltan is the Head of Mechatronics, Intelligent System, and Robotics Laboratory at the Iranian Institute of Advanced Science and Technology (IRAN SSP). Mr. Piltan led several high impact projects involving more than 150 researchers from countries around the world including Iran, Finland, Italy, Germany, South Korea, Australia, and the United States. Mr. Piltan has authored or coauthored more than 140 papers in academic journals, conference papers and book chapters. His papers have been cited at least 3900 times by independent and dependent researchers from around the world including Iran, Algeria, Pakistan, India, China, Malaysia, Egypt, Columbia, Canada, United Kingdom, Turkey, Taiwan, Japan, South Korea, Italy, France, Thailand, Brazil and more. Moreover, Mr. Piltan has peer-reviewed at least 23 manuscripts for respected international journals in his field. Mr. Piltan will also serve as a technical committee member of the upcoming EECSI 2015 Conference in Indonesia. Mr. Piltan has served as an editorial board member or journal reviewer of several international journals in his field as follows: International Journal Of Control And Automation (IJCA), Australia, ISSN: 2005-4297, International Journal of Intelligent System and Applications (IJISA), Hong Kong, ISSN:2074-9058, IAES International Journal Of Robotics And Automation, Malaysia, ISSN:2089-4856, International Journal of Reconfigurable and Embedded Systems, Malaysia, ISSN:20894864.

Mr. Piltan has acquired a formidable repertoire of knowledge and skills and established himself as one of the leading young scientists in his field. Specifically, he has accrued expertise in the design and implementation of intelligent controls in nonlinear systems. Mr. Piltan has employed his remarkable expertise in these areas to make outstanding contributions as detailed follows:Nonlinear control for industrial robot manipulator (2010IRAN SSP), Intelligent Tuning The Rate Of Fuel Ratio In Internal Combustion Engine (2011-IRANSSP), Design High Precision and Fast Dynamic Controller For Multi-Degrees Of Freedom Actuator (2013-IRANSSP), Research on Full Digital Control for Nonlinear Systems (2011-IRANSSP), Micro-Electronic Based Intelligent Nonlinear Controller (2015-IRANSSP), Active Robot Controller for Dental Automation (2015-IRANSSP), Design a Micro-Electronic Based Nonlinear Controller for First Order Delay System (2015-IRANSSP).

The above original accomplishments clearly demonstrate that Mr. Piltan has performed original research and that he has gained a distinguished reputation as an outstanding scientist in the field of electronics and control engineering. Mr. Piltan has a tremendous and unique set of skills, knowledge and background for his current and future work. He possesses a rare combination of academic knowledge and practical skills that are highly valuable for his work. In 2011, he published 28 first author papers, which constitute about $30 \%$ of papers published by the Department of Electrical and 
Electronic Engineering at University Putra Malaysia. Additionally, his 28 papers represent about $6.25 \%$ and $4.13 \%$ of all control and system papers published in Malaysia and Iran, respectively, in 2011.

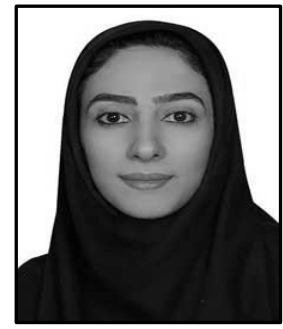

Maryam Sarostad is currently working as a research assistant in Control and Robotic Lab at the institute of advanced science and technology, IRAN SSP research and development Center. Her current research interest is in the area of system identification.

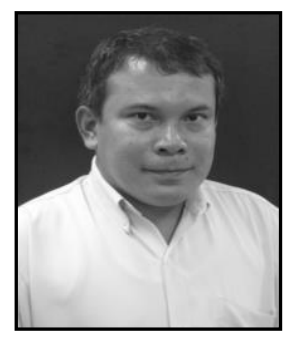

Nasri Sulaiman, is advisor and supervisor of several high impact projects involving more than 150 researchers from countries around the world including Iran, Malaysia, Finland, Italy, Germany, South Korea, Australia, and the United States. Dr. Nasri Sulaiman has authored or co-authored more than 80 papers in academic journals, conference papers and book chapters. His papers have been cited at least 3000 times by independent and dependent researchers from around the world including Iran, Algeria, Pakistan, India, China, Malaysia, Egypt, Columbia, Canada, United Kingdom, Turkey, Taiwan, Japan, South Korea, Italy, France, Thailand, Brazil and more. Dr. Nasri Sulaiman has employed his remarkable expertise in these areas to make outstanding contributions as detailed below:

- Design of a reconfigurable Fast Fourier Transform (FFT) Processor using multi-objective Genetic Algorithms (2008-UPM)

- Power consumption investigation in reconfigurable Fast Fourier Transform (FFT) processor (2010-UPM)

- Crest factor reduction And digital predistortion Implementation in Orthogonal frequency Division multiplexing (ofdm) systems (2011UPM)

- High Performance Hardware Implementation of a Multi-Objective Genetic Algorithm, (RUGS), Grant amount RM42,000.00, September (2012-UPM)

- Nonlinear control for industrial robot manipulator (2010-IRAN SSP)

- Intelligent Tuning The Rate Of Fuel Ratio In Internal Combustion Engine (2011-IRANSSP)

- Design High Precision and Fast Dynamic Controller For MultiDegrees Of Freedom Actuator (2013-IRANSSP)

- Research on Full Digital Control for Nonlinear Systems (2011IRANSSP)

- Micro-Electronic Based Intelligent Nonlinear Controller (2015IRANSSP)

- Active Robot Controller for Dental Automation (2015-IRANSSP)

- Design a Micro-Electronic Based Nonlinear Controller for First Order Delay System (2015-IRANSSP) 
International Journal of Smart Home

Vol. 11, No. 1 (2017) 